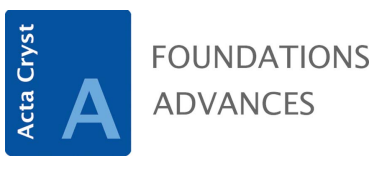

ISSN 2053-2733

\title{
Modern X-ray Analysis on Single Crystals. A Practical Guide. Second edition. By Peter Luger. De Gruyter, 2014. Pp. xi+334. Price EUR 119.95/USD 168.00/ GBP 89.99. ISBN 978-3-11-030823-5.
}

\author{
Claude Lecomte* \\ Université de Lorraine, CRM2, UMR 7036, Vandoeuvre-les-Nancy, F-54506, France, and CNRS, CRM2, UMR 7036, \\ Vandoeuvre-les-Nancy, F-54506, France. *Correspondence e-mail: claude.lecomte@crm2.uhp-nancy.fr
}

Keywords: book review; X-ray analysis; single crystals.

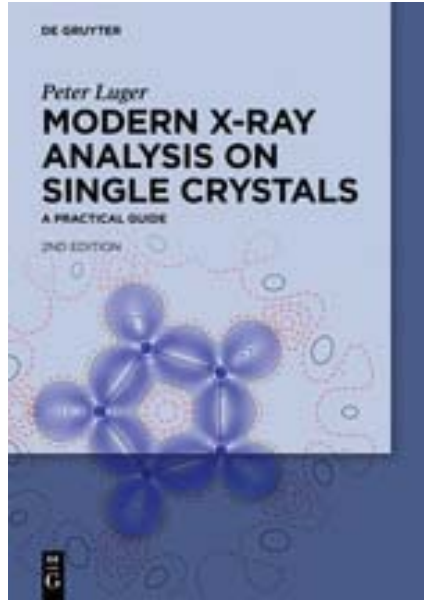

(C) 2016 International Union of Crystallography
The first edition of this book was widely recognized as one of the best X-ray crystallography books for anyone starting crystallography to learn organic/organometallic single-crystal structure determination. I have recommended this book to most of my students. The book was written in 1980 at the beginning of the automation of crystal structure determination at a time when it was really necessary to understand the basic theory. An excellent review of the book has been published by S. C. Wallwork (1981), andis still up to date for this second edition. Nowadays, modern diffractometers and efficient crystal structure determination and refinement packages allow one to determine most small-molecule crystal structures during one working day, but analysing the structures in terms of accuracy and intermolecular interactions, or solving difficult structures still require a solid theoretical background; reading the second edition of this book is an excellent start.

The major changes in the new edition are as follows:

The very good mathematical background chapter has been moved to an appendix which enables the reader to start directly with crystallography.

A critical description of X-ray micro-sources and synchrotron sources has been added. Professor Luger was one of the first small-molecule crystallographers to use synchrotron radiation for accurate data collection; he very well knows the pros and cons of such an experiment and I really regret that he did not develop more this important topic in the second edition of this book. It would have been much more useful than keeping a still extensive presentation of Weissenberg and precession methods, even though I understand that, for pedagogical reasons, the rotation method should be explained.

The theory on four-circle single-crystal diffractometers is mostly limited to point detector data collection with too little on area detectors, in particular: how to estimate the standard deviations on the intensities? How to estimate weak intensities? How do the black-box data-reduction programs, which the instrumentation companies provide, estimate $I(h, k, l)$ and its associated variance? How to collect really accurate data?

The chapter on least-squares refinement is very clear and useful for a beginner; it would have been interesting to comment more on the goodness of fit (GoF) and its relation to the intensity weighting; an important point missing is a discussion on the GoF at the end of an independent-atom crystal structure refinement, which should not be equal to 1 as the independent-atom model is wrong (see Chapter 9 devoted to electrondensity modelling).

Chapter 9 is a new chapter devoted to structure analysis under non-routine conditions which deals with: (i) low-temperature experiments, including neutron diffraction, and crystallization of low-melting-point compounds (applied to $\mathrm{NO}_{2}$ and $\beta$-borane), and (ii) structure analysis at high pressure and pressure cells.

Electron-density analysis is a speciality of Professor Luger. The kappa and multipole models are described together with applications to life science. This new part of the book is necessarily limited but is a very good introduction for readers who want to go beyond the spherical-atom model and the bibliography given is exhaustive. 


\section{book reviews}

In conclusion, this comprehensive book is very well written and illustrated; it is a book written by a colleague widely recognized in the crystallography community. I recommend it to all structural scientists who are starting out in crystallography and want to understand crystal structure determinations of organic and organometallic structures; it is also an excellent book for professional crystallographers.

\section{References}

Wallwork, S. C. (1981). Acta Cryst. A37, 270. 